This item was submitted to Loughborough's Research Repository by the author.

Items in Figshare are protected by copyright, with all rights reserved, unless otherwise indicated.

\title{
The existence of Rayleigh-Bloch surface waves
}

PLEASE CITE THE PUBLISHED VERSION

PUBLISHER

(c) Cambridge University Press

VERSION

VoR (Version of Record)

LICENCE

CC BY-NC-ND 4.0

REPOSITORY RECORD

Linton, C.M., and M. Mclver. 2019. "The Existence of Rayleigh-bloch Surface Waves". figshare. https://hdl.handle.net/2134/4429. 
This item was submitted to Loughborough's Institutional Repository (https://dspace.lboro.ac.uk/) by the author and is made available under the following Creative Commons Licence conditions.

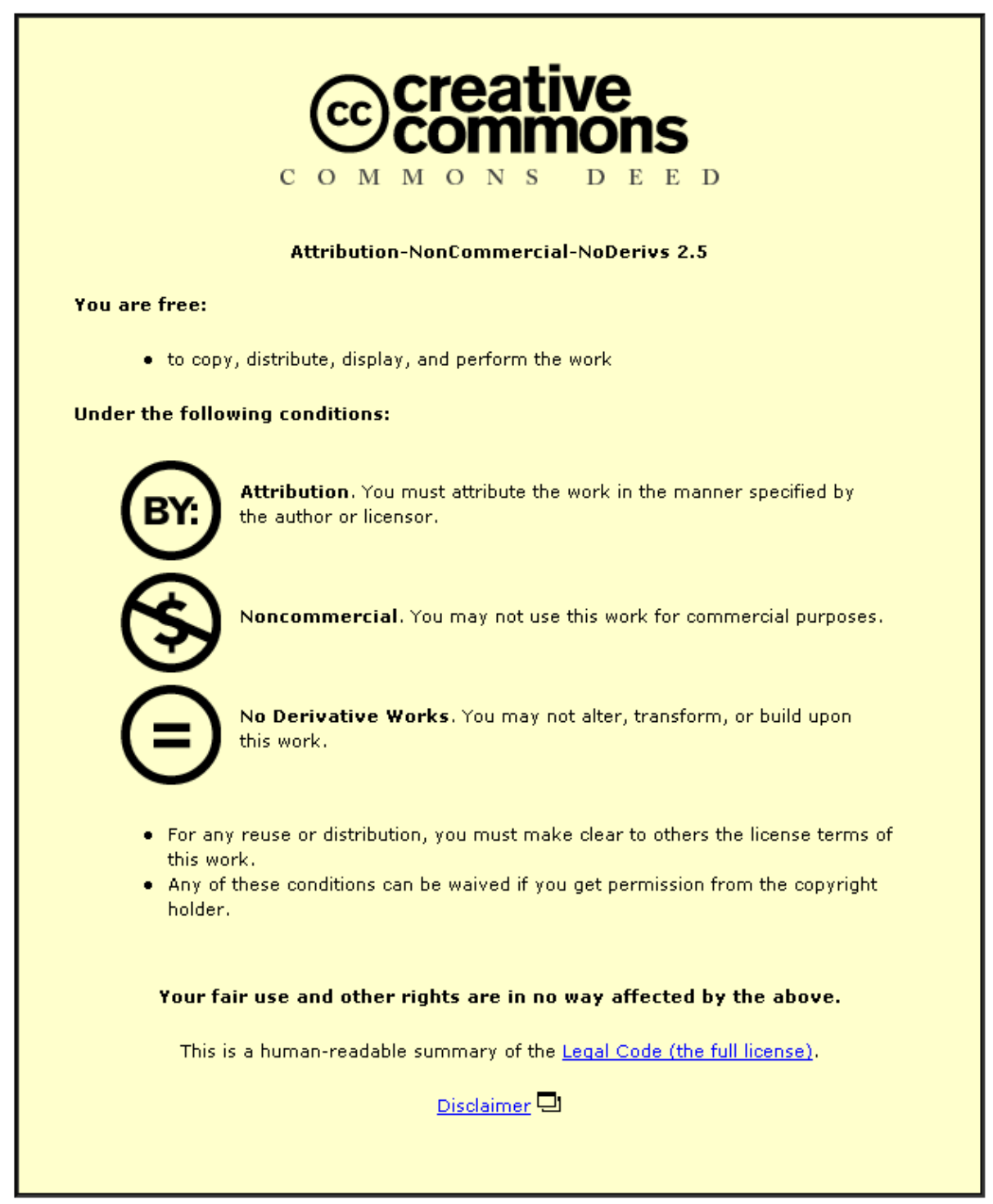

For the full text of this licence, please go to: http://creativecommons.org/licenses/by-nc-nd/2.5/ 


\title{
The existence of Rayleigh-Bloch surface waves
}

\author{
By C. M. LINTON AND M. McIVER \\ Department of Mathematical Sciences, Loughborough University, \\ Leicestershire LE11 3TU, UK \\ (Received 8 May 2002 and in revised form 6 August 2002)
}

Rayleigh-Bloch surface waves arise in many physical contexts including water waves and acoustics. They represent disturbances travelling along an infinite periodic structure. In the absence of any existence results, a number of authors have previously computed such modes for certain specific geometries. Here we prove that such waves can exist in the absence of any incident wave forcing for a wide class of structures.

\section{Introduction}

We are concerned here with the existence of waves propagating along a diffraction grating in two dimensions, in the absence of any incident wave field. Specifically, we consider a grating in the form of a periodic continuous curve $\Gamma$ of the form $x=g(y)$, where $g(y)$ has period $d$ and $|g(y)|$ is bounded. (We allow discontinuities in $g(y)$, in which case $\Gamma$ is assumed to be parallel to the $x$-axis at a point of discontinuity.) We then ask whether non-trivial solutions to the Helmholtz equation $\left(\nabla^{2}+k^{2}\right) \phi=0$ exist in $x>g(y)$, which satisfy homogeneous Neumann or Dirichlet boundary conditions on $\Gamma$, and which decay exponentially as $x \rightarrow \infty$. Such solutions are known as pure Rayleigh-Bloch surface waves. The values of $k^{2}$ at which they occur are eigenvalues of the operator $-\nabla^{2}$ subject to the appropriate boundary conditions.

This problem derives from the study of acoustic and electromagnetic waves, and also arises in the analysis of water waves propagating along periodic coastlines in an ocean of constant depth (in which case the two-dimensional Helmholtz equation arises after factoring out the depth dependence). In this latter context these disturbances correspond to edge waves travelling parallel to the shoreline while decaying out to sea, but in contrast to the more usual type of edge wave they are supported by the shape of the coastline rather than any variation in depth as one moves away from the shore. They also represent waves propagating along a periodic array of vertical cylinders of constant cross-section.

No general existence result appears to be available if the boundary condition on $\Gamma$ is of Neumann type, though in Bonnet-Bendhia \& Starling (1994) (hereafter referred to as BBS) it was proved that there are geometries for which pure Rayleigh-Bloch surface waves do exist. To the authors' knowledge, no general criteria for whether a given geometry supports such waves have been determined. Furthermore, such modes have been observed experimentally (Barlow \& Karbowiak 1954) and computed numerically for many geometries (see Porter \& Evans 1999, and the references cited therein). It should be noted that BBS treat the more general problem in which the boundary $\Gamma$ is contained within a periodic dielectric medium. The existence of surface waves for diffraction gratings consisting of a periodic dielectric strip (of constant width) on a flat boundary (either Neumann or Dirichlet) is discussed in Grikurov et al. (2000).

The solutions which have been computed have a dominant wavenumber $\beta$ and this 
acts as a cut-off for the problem, in that for $k^{2}<\beta^{2}$ waves cannot propagate away from the grating. This allows us to use a standard variational approach to search for eigenvalues $k^{2}$ in the interval $\left(0, \beta^{2}\right)$. The analytic framework for this problem has been set up in BBS, and in this note we simply construct suitable test functions which can be used to prove the existence of such eigenvalues for a wide class of diffraction gratings, using the techniques described in Evans, Levitin \& Vassiliev (1994).

If the boundary condition on $\Gamma$ is of Dirichlet type, it is known that such solutions do not exist for $k^{2}<\beta^{2}$ (see, for example, BBS, Theorem 4.1).

\section{Proof of existence}

Let $\Omega$ be the domain $\{(x, y): x>g(y), 0<y<d\}$. Let $H_{\beta}^{1}(\Omega)$ be the space of functions $\left\{\phi \in L^{2}(\Omega),|\nabla \phi| \in L^{2}(\Omega)\right\}$ which also satisfy the boundary conditions

$$
\left.\phi\right|_{y=d}=\left.\mathrm{e}^{\mathrm{i} \beta d} \phi\right|_{y=0},\left.\quad \frac{\partial \phi}{\partial y}\right|_{y=d}=\left.\mathrm{e}^{\mathrm{i} \beta d} \frac{\partial \phi}{\partial y}\right|_{y=0} .
$$

It is evident that we only need to consider $\beta d$ in an interval of length $2 \pi$ and it turns out that it is convenient to restrict $\beta d$ to lie in the interval $(-\pi, \pi]$. The space $H_{\beta, 0}^{1}(\Omega)$ is the subspace of $H_{\beta}^{1}(\Omega)$ which consists of those functions which also satisfy $\phi=0$ on $\Gamma$.

We denote by $A_{\mathrm{N}}$ (resp. $A_{\mathrm{D}}$ ) the positive self-adjoint operator $-\nabla^{2}$ on $\Omega$ restricted to functions in $H_{\beta}^{1}(\Omega)$ (resp. $H_{\beta, 0}^{1}(\Omega)$ ). Our aim is to establish whether $A_{\mathrm{N}}$ has any eigenvalues. The spectrum of an operator $A, \sigma(A)$, (i.e. the set containing all the values of $k^{2}$ for which $A-k^{2} I$ does not have a bounded inverse) is made up of the discrete spectrum, containing any isolated eigenvalues of finite multiplicity, and the essential spectrum, $\sigma_{\text {ess }}(A)$. It is proved in BBS that

$$
\sigma_{\text {ess }}\left(A_{\mathrm{N}}\right)=\sigma\left(A_{\mathrm{D}}\right)=\left[\beta^{2}, \infty\right) .
$$

It immediately follows that there are no eigenvalues $k^{2}<\beta^{2}$ for the Dirichlet problem. From the variational formulation of the problem set up in BBS we also know that the lowest point of the spectrum of $A_{\mathrm{N}}$ is inf $Q[\psi]$, where the Rayleigh quotient $Q[\psi]$ is given by

$$
Q[\psi]=\frac{\int_{\Omega}|\nabla \psi|^{2} \mathrm{~d} \Omega}{\int_{\Omega}|\psi|^{2} \mathrm{~d} \Omega}
$$

and the infimum is taken over all functions in $H_{\beta}^{1}(\Omega) \backslash\{0\}$. Thus if we can find a function $\psi \in H_{\beta}^{1}(\Omega) \backslash\{0\}$ for which $Q[\psi]<\beta^{2}$, then an eigenvalue $k^{2}<\beta^{2}$ must exist.

The boundary curve $\Gamma$ is of the form $x=g(y)$ where $g(y)$ has period $d$ and it is assumed that $\Gamma$ has a well-defined normal at all but a finite number of points so that it makes sense to apply the boundary condition

$$
\frac{\partial \phi}{\partial n}=0 \text { on } \Gamma .
$$

The results in (2.2) rely on the fact that if the domain $\Omega$ were truncated at $x=X$ then the spectrum of the Laplacian would be entirely discrete. To ensure that this is the case we assume that $\Omega$ has no outward pointing cusps (i.e. the boundary $\Gamma$ has no inward pointing cusps). We will assume that the line $x=0$ is positioned so that $\max g(y)=-\min g(y)=a$, say (the maximum value may be attained more than once 


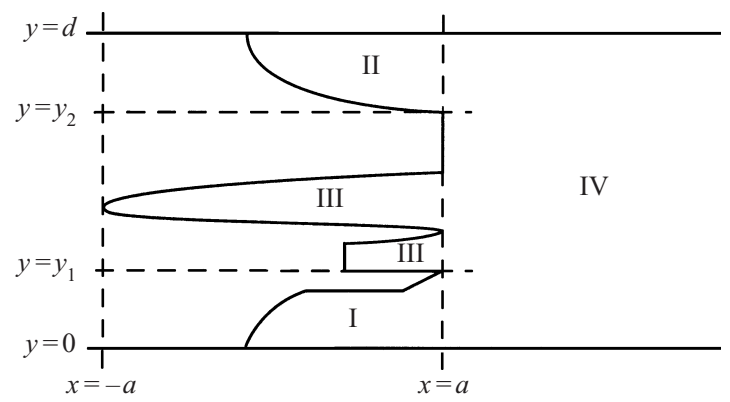

FigURE 1. First type of geometry under consideration.

in any one period of $g$ ). Let $y_{1}$ be the smallest value of $y$ in $[0, d]$ for which $g(y)=a$ and let $y_{2}$ be the largest such value. Clearly $0 \leqslant y_{1} \leqslant y_{2} \leqslant d$. For a given periodic boundary, excluding the case $g(y) \equiv 0$, we can always choose axes so that

$$
0<y_{1}=d-y_{2}<d \text {. }
$$

We then divide the domain $\Omega$ into four regions. Region I is $\left\{0<y<y_{1}, g(y)<x<a\right\}$, region II is $\left\{y_{2}<y<d, g(y)<x<a\right\}$, region III is $\left\{y_{1}<y<y_{2}, g(y)<x<a\right\}$, and region IV is $\{0<y<d, a<x\}$. Region III may be disconnected and may of course be empty. These remarks are illustrated in figure 1 in which the geometry shown is intended to demonstrate the various possibilities, rather than represent any realistic situation. Geometries of this type include the 'comb' grating considered by Evans \& Linton (1993), the array of rectangular protrusions treated in Evans \& Fernyhough (1995), and an infinite array of circles (for which Rayleigh-Bloch modes were constructed in McIver, Linton \& McIver 1998) since the line of symmetry of the array can be treated as a Neumann boundary.

We introduce a positive parameter $\epsilon$ and define our test function by

$$
\psi= \begin{cases}\mathrm{e}^{\mathrm{i} \beta y}+\epsilon^{1 / 2} \cos (\pi x / 2 a) & \text { in I } \\ \mathrm{e}^{\mathrm{i} \beta y}+\epsilon^{1 / 2} \mathrm{e}^{\mathrm{i} \beta d} \cos (\pi x / 2 a) & \text { in II } \\ \mathrm{e}^{\mathrm{i} \beta y} & \text { in III } \\ \mathrm{e}^{\mathrm{i} \beta y} \mathrm{e}^{-\epsilon(x-a)} & \text { in IV. }\end{cases}
$$

This function satisfies (2.1), is continuous throughout the fluid domain, and tends to zero exponentially as $x \rightarrow \infty$. It thus belongs to $H_{\beta}^{1}(\Omega) \backslash\{0\}$. A straightforward calculation reveals that, for $\beta \neq 0$,

$$
\begin{aligned}
\beta^{-2} Q[\psi] & =\frac{\frac{1}{2} d \epsilon^{-1}+\int_{0}^{d}[a-g(y)] \mathrm{d} y+O(\epsilon)}{\frac{1}{2} d \epsilon^{-1}+\int_{0}^{d}[a-g(y)] \mathrm{d} y+4 d \epsilon^{1 / 2} J+O(\epsilon)} \\
& =1-8 \epsilon^{3 / 2} J+O\left(\epsilon^{2}\right),
\end{aligned}
$$

where

$$
J=\frac{a}{\pi d} \int_{0}^{y_{1}}\left(2-\sin \frac{\pi g(y)}{2 a}-\sin \frac{\pi g(d-y)}{2 a}\right) \cos \beta y \mathrm{~d} y .
$$

The integrand in this expression is strictly positive on $\left(0, y_{1}\right)$ since $y_{1} \leqslant \frac{1}{2} d,|\beta d| \leqslant \pi$, 


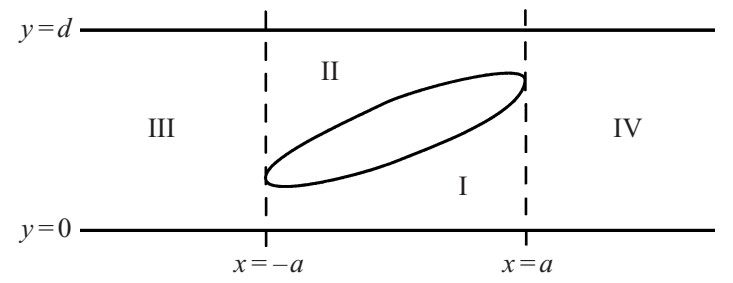

FIGURE 2. Second type of geometry under consideration.

and both $|g(y)|$ and $|g(d-y)|$ are strictly less than $a$ for $y \in\left(0, y_{1}\right)$. Thus $J>0$ and $\epsilon$ can be chosen sufficiently small so that $Q[\psi]<\beta^{2}$. Of course, in the limit as $a \rightarrow 0$ we have $J \rightarrow 0$ and the existence argument breaks down, as one would expect since there are no Rayleigh-Bloch surface waves if the boundary $\Gamma$ is a straight line.

Some remarks concerning the particular form chosen for the test function are appropriate. First, as $\epsilon \rightarrow 0$, the test function approaches $\exp (i \beta y)$ and $Q[\psi]$ tends to the cut-off $\beta^{2}$. This is essential if it is going to be possible to find an $\epsilon$ for which $Q[\psi]<\beta^{2}$ for any geometry of the type shown in figure 1 , since geometries can be found for which the eigenvalue $k^{2}$ is as close as we please to $\beta^{2}$. For a specific geometry our test function $\psi$ yields an upper bound, $Q[\psi]$, for the eigenvalue $k^{2}$, but there is no reason why this should be a good approximation. The most obvious test function to try would be simply given by $\exp (\mathrm{i} \beta y)$ in I, II and III, rather than the form given in (2.6). This would then be of the same type as the test function used by Evans et al. (1994), but it turns out that this does not work for our problem, since $Q[\psi]=\beta^{2}+O\left(\epsilon^{2}\right)$ as $\epsilon \rightarrow 0$. In order to remedy the situation we have modified this basic test function by making suitable small changes to $\psi$ in regions I and II in such a way that $\psi$ still satisfies the periodicity conditions (2.1) and is still continuous across $x=a$. There is no unique way of doing this; the form given above is merely the simplest that we have been able to construct (note that the additional terms in regions I and II could contain $\epsilon^{q}$ for any $q \in(0,1)$, there is no need for $q$ to be $\frac{1}{2}$ ).

This completes the existence proof for the geometries described above, which includes some, but not all, of the geometries for which pure Rayleigh-Bloch modes have been constructed numerically. For example, it does not include the infinite array of staggered plates considered by Koch (1983), nor the angled ellipses treated in Porter \& Evans (1999). Existence can be proved for these and similar cases as follows.

Consider a periodic array of closed contours, each one defined by two curves $y=f_{1}(x)$ and $y=f_{2}(x)\left(0<f_{1}(x) \leqslant f_{2}(x)<d\right)$ as illustrated in figure 2 . As in the previous case, we assume that the boundary has a well-defined normal at all but a finite number of points but we allow discontinuities in $f_{1}$ and $f_{2}$, in which case the boundary is assumed to be parallel to the $y$-axis at a point of discontinuity.

The fluid domain is again divided into four regions as shown in figure 2 and we use the test function

$$
\psi= \begin{cases}\mathrm{e}^{\mathrm{i} \beta y}+\epsilon^{1 / 2} \cos (\pi x / 2 a) & \text { in I } \\ \mathrm{e}^{\mathrm{i} \beta y}+\epsilon^{1 / 2} \mathrm{e}^{\mathrm{i} \beta d} \cos (\pi x / 2 a) & \text { in II } \\ \mathrm{e}^{\mathrm{i} \beta y} \mathrm{e}^{\epsilon(x+a)} & \text { in III } \\ \mathrm{e}^{\mathrm{i} \beta y} \mathrm{e}^{-\epsilon(x-a)} & \text { in IV }\end{cases}
$$




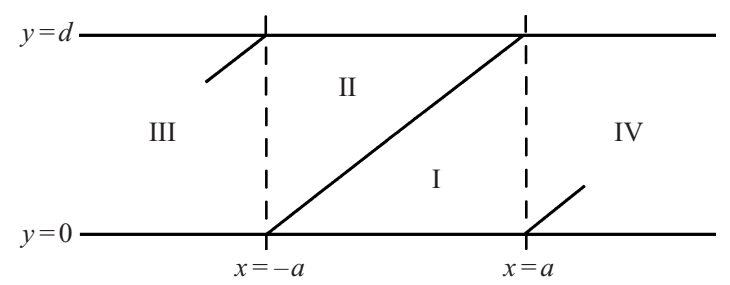

FIGURE 3. One period of an overlapping staggered-plate array.

and find that, for $\beta \neq 0$,

$$
\beta^{-2} Q[\psi]=1-2 \epsilon^{3 / 2} J+O\left(\epsilon^{2}\right)
$$

where

$$
J=\frac{1}{\beta d} \int_{-a}^{a} \cos \frac{\pi x}{2 a}\left(\sin \left[\beta f_{1}(x)\right]+\sin \left[\beta\left(d-f_{2}(x)\right)\right]\right) \mathrm{d} x .
$$

Since $|\beta d| \leqslant \pi$, the integrand is strictly positive for $x \in(-a, a)$ and so $J>0$. Hence $\epsilon$ can be chosen sufficiently small so that $Q[\psi]<\beta^{2}$ in this case also.

The staggered-plate arrays treated by Koch (1983) are not covered by geometries of the above type because the plates overlap so that each period contains sections from more than one plate in the array. However, such situations are easily included by defining $a$ to be the maximum value which allows just one plate in the region $|x|<a, 0<y<d$, as shown in figure 3 . We can then proceed exactly as in the previous example, the presence of parts of the geometry in regions I and IV making no difference.

\section{Conclusion}

We have proved that pure Rayleigh-Bloch surface waves exist for an extremely wide class of periodic geometries, including all those for which strong numerical evidence has previously been presented. In the water wave context our results establish the existence of edge waves travelling along general periodic coastlines and along periodic arrays of cylinders of arbitrary cross-section. We have not attempted to characterize the most general form of geometry for which modes exist, however, and some generalizations are obvious. For example, in the any of the examples above we can clearly add further closed contours within regions I and II.

It is clear from our results that Rayleigh-Bloch surface waves do exist, in the absence of any incident wave forcing, for the vast majority of periodic structures of practical significance. Indeed, other than the degenerate case of a straight boundary, we know of no periodic structures which do not support such waves.

The authors would like to thank Dr M. D. Groves for his useful comments.

\section{REFERENCES}

Barlow, H. E. M. \& Karbowiak, A. E. 1954 An experimental investigation of the properties of corrugated cylindrical surface waveguides. Proc. IEE 101 (Part III), 182-188.

Bonnet-Bendhia, A.-S. \& Starling, F. 1994 Guided waves by electromagnetic gratings and nonuniqueness examples for the diffraction problem. Math. Meth. Appl. Sci. 17, 305-338.

Evans, D. V. \& Fernyhough, M. 1995 Edge waves along periodic coastlines. Part 2. J. Fluid Mech. 297, 301-325. 
Evans, D. V., Levitin, M. \& Vassiliev, D. 1994 Existence theorems for trapped modes. J. Fluid Mech. 261, 21-31.

Evans, D. V. \& Linton, C. M. 1993 Edge waves along periodic coastlines. Q. J. Mech. Appl. Maths 46, 642-656.

Grikurov, V. E., Lyalinov, M. A., NeittaanmäKi, P. \& Plamenevskit, B. A. 2000 On surface waves in diffraction gratings. Math. Meth. Appl. Sci. 23, 1513-1535.

Koch, W. 1983 Resonant acoustic frequencies of flat plate cascades. J. Sound Vib. 88, 233-242.

McIver, P., Linton, C. M. \& McIver, M. 1998 Construction of trapped modes for wave guides and diffraction gratings. Proc. R. Soc. Lond. A 454, 2593-2616.

Porter, R. \& Evans, D. V. 1999 Rayleigh-Bloch surface waves along periodic gratings and their connection with trapped modes in waveguides. J. Fluid Mech. 386, 233-258. 